\title{
Chave de identificação dos Ctenophora da costa brasileira
}

\author{
Otto Müller Patrão de Oliveira ${ }^{1,2,5}$, Hermes Mianzan ${ }^{3,4}$, \\ Alvaro Esteves Migotto $^{2}$ \& Antonio Carlos Marques ${ }^{1}$ \\ Biota Neotropica $v 7$ (n3) \\ http://www.biotaneotropica.org.br/v7n3/pt/abstract?identification-key+bn03507032007 \\ Recebido em 13/06/07 \\ Versão reformulada recebida em 21/09/07 \\ Publicado em 22/10/07 \\ ${ }^{1}$ Departamento de Zoologia, Instituto de Biociências, Universidade de São Paulo - USP, \\ R. Matão, Trav. 14, 101, CEP 05508-900, São Paulo, SP, Brasil \\ ${ }^{2}$ Centro de Biologia Marinha, Universidade de São Paulo - USP, \\ Rodovia Manoel H. do Rego Km 131,5, CEP 11600-000, São Sebastião, SP, Brasil \\ ${ }^{3}$ Instituto Nacional de Investigación y Desarrollo Pesquero, \\ Paseo V. Ocampo, 1, B7602HSA, Mar del Plata, Argentina \\ ${ }^{4}$ Consejo Nacional de Investigaciones Científicas y Técnicas, Rivadavia 1917, Buenos Aires, Argentina \\ ${ }^{5}$ Autor para correspondência: Otto Müller Patrão de Oliveira, e-mail: ottompo@usp.br
}

\begin{abstract}
Oliveira, O.M.P., Mianzan, H., Migotto, A.E. \& Marques, A.C. Identification key for the ctenophores from Brazilian coast. Biota Neotrop. Sep/Dez 2007 vol. 7, no. 3 http://www.biotaneotropica.org.br/v7n3/pt/ abstract?identification-key+bn03507032007. ISSN 1676-0603.

Although ctenophores are abundant and ecologically important in the marine environment, they are poorly known in the Brazilian coast. The present study is a taxonomic key for the ctenophores from the Brazilian coast. It aims to help students and non-specialist researchers with the identification of those organisms. Collecting, preserving and photographing methods are described. Characters that distinguish the thirteen species registered
\end{abstract} in Brazilian marine territories are presented.

Keywords: Ctenophora, comb-jelly, zooplankton, gelatinous plankton, pelagial, benthos, Brazil.

\section{Resumo}

Oliveira, O.M.P., Mianzan, H., Migotto, A.E. \& Marques, A.C. Chave de identificação dos Ctenophora da costa brasileira. Biota Neotrop. Sep/Dez 2007 vol. 7, no. 3 http://www.biotaneotropica.org.br/v7n3/pt/ abstract?identification-key+bn03507032007. ISSN 1676-0603.

Embora abundantes e importantes ecologicamente no meio marinho, os ctenóforos do litoral brasileiro têm sido pouco estudados. O presente estudo tem por objetivo prover informações para auxiliar na identificação desses organismos. Para tal, são descritos métodos de fixação e documentação fotográfica dos ctenóforos. A terminologia referente ao grupo, em língua portuguesa, é apresentada na forma de um glossário. Além disso, as características que distinguem as treze espécies registradas para águas brasileiras são organizadas em uma chave de identificação. A complementação da identificação pode ser feita pela literatura indicada para cada espécie.

Palavras-chave: ctenóforos, zooplâncton, plâncton gelatinoso, pelagial, bentos, Brasil. 


\section{Introdução}

Os Ctenophora são um filo de animais marinhos de corpo translúcido, gelatinoso e frágil. Caracteristicamente apresentam, pelo menos em alguma fase do seu ciclo de vida, oito fileiras longitudinais de ctenos (pentes formados por cílios justapostos) que, em geral, atuam na locomoção (Harbison \& Madin, 1982).

Devido à simetria corpórea birradial (Figura 1), o grupo já foi incluído entre os Radiata, juntamente com cnidários e equinodermos (L. Agassiz 1860). Posteriormente, foi considerado como parte dos Coelenterata, por compartilhar com os Cnidaria características como a constituição gelatinosa do corpo e ausência de um sistema digestivo completo (Hyman 1940). Atualmente, a posição dos Ctenophora na filogenia dos Metazoa é incerta. Embora estudos morfológicos os coloquem como grupo irmão de todos os animais bilaterais (e.g. Nielsen 2001, Brusca \& Brusca 2007), dados moleculares são inconclusivos, oscilando sua posição entre os filos basais (e.g. Zrzavý et al. 1998, Podar et al. 2001, Wallberg et al. 2004).

Embora seja um grupo com relativamente poucas espécies (cerca de 120, segundo Mills 1998-2007), estas são geralmente conspícuas, abundantes e de ampla distribuição. Os Ctenophora são mais conhecidos pelas belas formas planctônicas, mas há espécies bentônicas de hábitos crípticos e corpos vermiformes (Oliveira \& Migotto 2007). Ainda que as espécies planctônicas sejam quase transparentes, a bioluminescência (Haddock \& Case 1995) e a iridescência nos pentes ciliares (Welch et al. 2005) são comuns, propriedades que os tornam visíveis na coluna de água, tanto de noite quanto de dia.

Como vorazes predadores, esses animais ingerem grandes quantidades de pequenos organismos zooplanctônicos (Bishop 1968), incluindo larvas de peixes de grande importância econômica (Oliveira \& Marques 2007). Entretanto, alguns grupos de ctenóforos, como os Haeckeliidae e os Beroidae, se alimentam de outros organismos planctônicos gelatinosos, por vezes até maiores do que eles próprios
(Swanberg 1974, Mills \& Miller 1984). Sua atuação predatória, aliada ao rápido processo reprodutivo de algumas espécies, pode gerar problemas econômicos de larga escala, como no caso da introdução acidental de Mnemiopsis leidyi no Mar Negro, no início da década de 1980, transportado em água de lastro de navios (GESAMP 1997). Ao encontrar um ambiente favorável, desprovido de predadores, M. leidyi atingiu níveis extremos de superpopulação em poucos anos, reduzindo os estoques de peixes de importância comercial devido à predação sobre seus ovos e larvas, e sobre o plâncton, fonte de alimentação destes peixes (GESAMP 1997, Kideys 2002). Por outro lado, Beroe ovata foi também introduzido acidentalmente no Mar Negro. Este, devido ao hábito de se alimentar vorazmente de outros ctenóforos, foi o responsável pelo controle populacional do M. leidyi na região (Kideys 2002). Acredita-se que espécies do gênero Beroe sejam soluções para o controle da superpopulação de M. leidyi em outras áreas invadidas, como o Mar Cáspio (Kideys 2002), Mar Báltico (Oliveira 2007) e Mar do Norte (Boersma et al. 2007).

A maioria dos ctenóforos é hermafrodita simultâneo (Harbison \& Miller 1986), sendo geralmente capazes de auto-fecundação e, em alguns casos, iniciando o período reprodutivo já na fase larval (Baker \& Reeve 1974). Geralmente liberados no meio circundante, os ovos dão origem a uma larva, denominada cidipídia, exceto nos Beroidae, que possuem larvas morfologicamente semelhantes aos adultos (Harbison \& Madin 1982). Algumas espécies de Platyctenida (ordem de ctenóforos bentônicos) apresentam protandria (Harbison \& Madin 1982). A reprodução assexuada, pela fissão do corpo parental, também pode ocasionalmente ocorrer nos Platyctenida (Harbison \& Madin 1982).

Dentre as cerca de 120 espécies conhecidas atualmente (Mills 1998-2007), treze (vide tabela 1) foram reportadas em águas brasileiras (Marcus 1957, Mianzan 1999, Oliveira \& Migotto 2006). As dificuldades inerentes aos processos de coleta, preservação e

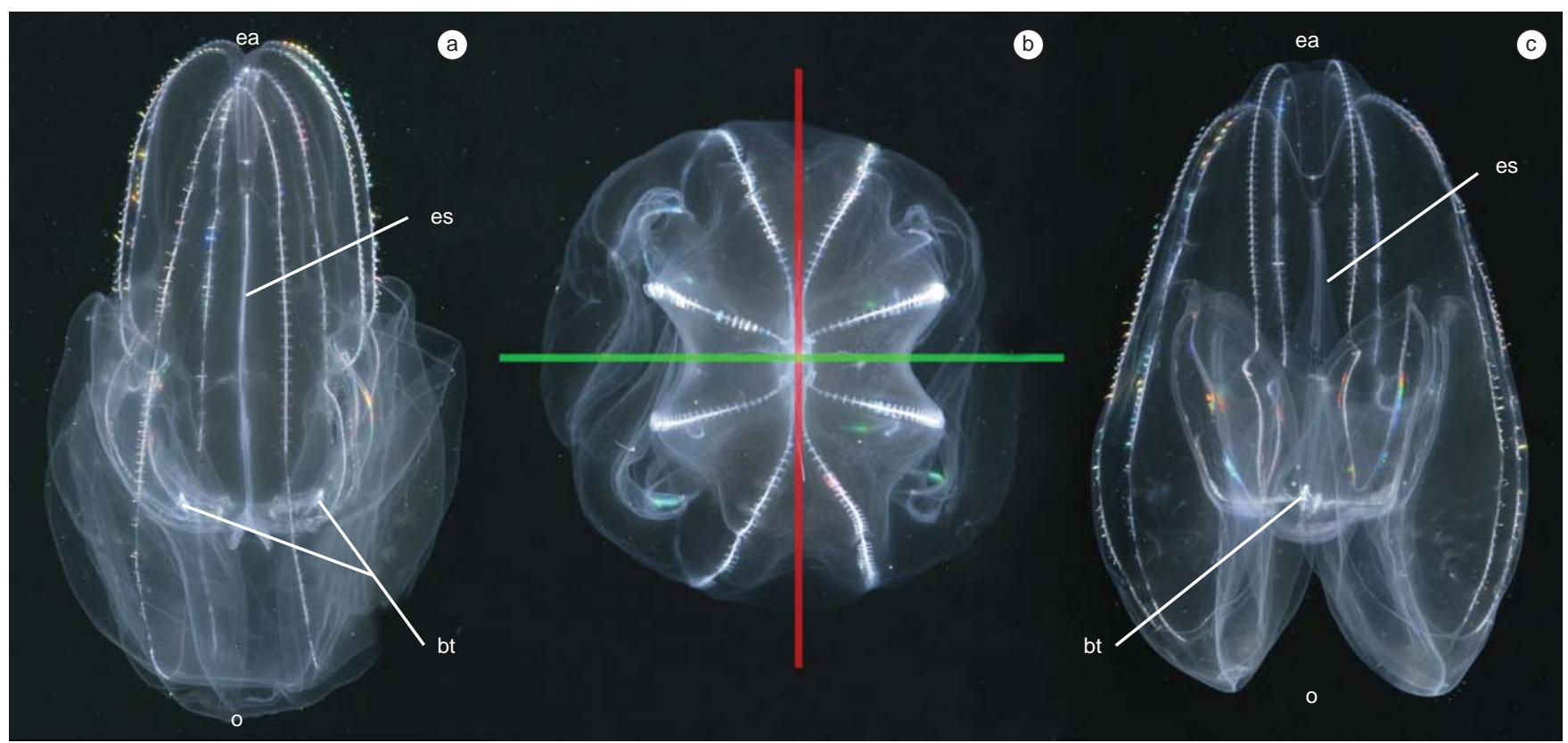

Figura 1. Representação dos planos de simetria dos ctenóforos, utilizando Bolinopsis vitrea como modelo. a) vista no plano tentacular; b) vista aboral mostrando os planos de corte no eixo estomodeal (em vermelho) e no eixo tentacular (em verde); e c) vista no plano estomodeal. (fotos: A. Migotto) Legenda: ea, extremidade aboral; bt, bainha tentacular; es, estomodeu; o, extremidade oral.

Figure 1. Symmetry planes of the ctenophores, using Bolinopsis vitrea as model. a) view of the tentacular plane; b) aboral view, showing the stomodeal axis (in red) and the tentacular axis (in green); and c) view of the stomodeal plane. (photos: A. Migotto) Legends: ea, aboral extremity; bt, tentacular bulb; es, stomodeum; o, oral extremity. 
Tabela 1. Quadro sinóptico da classificação dos ctenóforos encontrados na costa brasileira.

Table 1. Taxonomic classification of the ctenophores from the Brazilian coast.

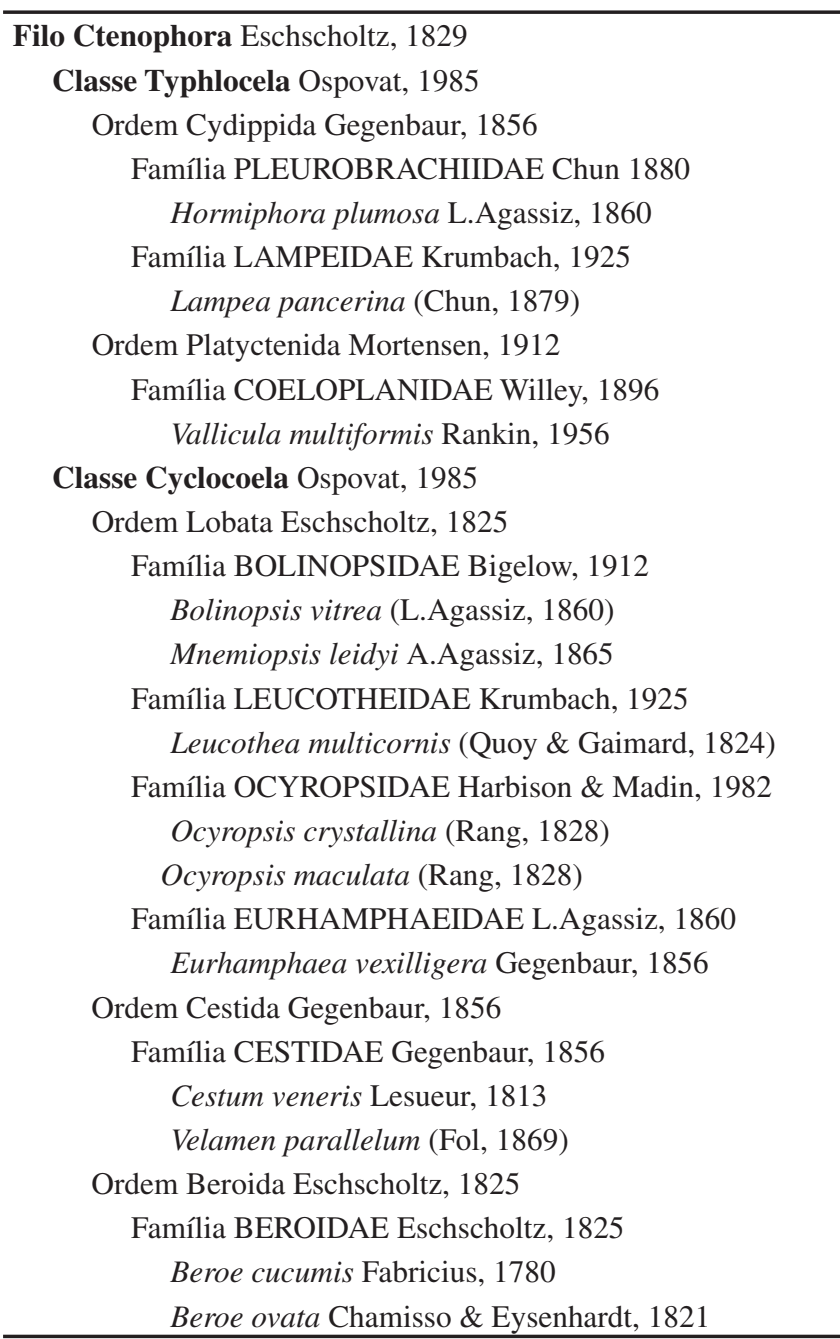

manutenção in vivo de espécimes têm sido responsáveis pelo parco conhecimento que se detém deste grupo animal até o momento.

O presente estudo tem por objetivo apresentar características que facilitem a identificação das espécies encontradas na costa brasileira, permitindo que estudantes e pesquisadores não familiarizados com o grupo possam fazer identificações confiáveis. Além disso, buscamos padronizar a terminologia técnica, em língua portuguesa, apresentando um glossário de termos comumente utilizados no estudo dos Ctenophora.

\section{Material e Métodos}

Embora atualmente o conhecimento dos ctenóforos tenha se beneficiado das modernas técnicas de observação e amostragem utilizando submersíveis tripulados ou não, a coleta por meios tradicionais é ainda o único recurso para os pesquisadores e instituições que não dispõem desses equipamentos de custo e operação elevados (Haddock 2004). A coleta de ctenóforos pelágicos pode ser feita por meio direto (em mergulho livre ou autônomo, veja Haddock \& Heine 2005) ou indireto (com a utilização de rede de plâncton). Embora a primeira (utilizando-se sacos ou recipientes plásticos) seja a preferen- cial, pois é a técnica que menos danifica esses frágeis organismos, ela não é viável em muitas situações, além de não ser adequada à coleta de exemplares pequenos (menos de $30 \mathrm{~mm}$ aproximadamente). A utilização de redes de plâncton é a opção geralmente mais simples. A rede deve ter malha variando entre 300 e $500 \mu \mathrm{m}$, boca larga $(60 \mathrm{~cm}$ de diâmetro ou mais) e copo com volume de pelo menos um litro. Os arrastos devem ser de curta duração (cerca de 5 minutos no máximo) e lentos, para evitar adensamento excessivo. $\mathrm{O}$ transporte dos espécimes durante as coletas deve ser feito, preferencialmente, em recipientes térmicos, com paredes arredondadas e com grandes volumes de água (pelo menos 10 vezes o volume dos animais coletados). As técnicas gerais de coleta, manutenção em laboratório e fixação são semelhantes às empregadas para outros organismos gelatinosos planctônicos, conforme detalhadas em Raskoff et al. (2003).

Ctenóforos bentônicos (no caso V. multiformis) são coletados examinando-se substratos diversos sob estereomicroscópio, tais como algas, conchas e seixos. Durante o transporte e triagem, é importante não deixar o material exposto ao ar, mantendo-o em um recipiente com a água do ambiente aonde foi coletado.

A fixação de ctenóforos nos meios fixadores comuns (formol e etanol) geralmente não produz bons resultados. Soluções fixadoras alternativas, como as de Adams et al. (1976), Corrêa (1987) e Mianzan (1999), além de serem tóxicas, também não produzem resultados plenamente satisfatórios, em especial a longo prazo. Dentre as espécies apresentadas neste estudo, apenas as do gênero Beroe conservam-se bem em formol. Os exemplares devem ser fixados em uma solução tamponada de formol a 5\% em água do mar. É importante medir o comprimento do animal antes da fixação e incluir esse dado na etiqueta de identificação, pois é esperado que os espécimes encolham gradual e intensamente após a fixação.

Para estudos bio-moleculares, o etanol a $95 \%$ é o fixador ideal. No etanol, a porção gelatinosa do animal extravasa do corpo, turvando a solução fixadora. Para acelerar o processo de extravasamento da mesogléia, pode-se agitar o frasco logo após a colocação do animal no fixador. O resíduo de material que fica no fundo do frasco são tecidos, justamente a porção que pode então ser utilizada para a extração de ácidos nucléicos.

Devido à impossibilidade de se preservar adequadamente os espécimes, a fotografia é uma ferramenta importante para a identificação específica ou mesmo como registro científico de sua ocorrência ("voucher specimen"). Fotografias subaquáticas dificilmente permitem a identificação no nível específico, porém têm a vantagem de retratar o animal em seu próprio ambiente. $\mathrm{O}$ mais indicado, todavia, é fotografar o ctenóforo vivo em laboratório. O microscópio óptico é ideal para fotografar larvas menores que $500 \mu \mathrm{m}$, preparadas em lâminas escavadas. Detalhes microscópicos, como os coloblastos, também devem ser fotografados no microscópio óptico, preparados entre lâmina e lamínula. Já o estereomicroscópio é indicado para fotografar espécimes inteiros, menores que $30 \mathrm{~mm}$, ou detalhes de espécies maiores. Ctenóforos maiores que $30 \mathrm{~mm}$ podem ser fotografados em aquário. É importante que o aquário seja montando em um ambiente escuro, para evitar reflexos, e sua parede traseira deve ser negra e não reflexiva, para proporcionar contraste adequado entre o fundo e o animal. O vidro frontal deve ser liso, livre de riscos e sujeiras, e a água do aquário deve ser filtrada. A câmara ideal deve ter lente macro, que deve ser mantida paralelamente ao vidro frontal do aquário. A fonte de iluminação artificial, com um ou dois flashes eletrônicos, deve ser colocada acima ou lateralmente ao aquário (veja Wrobel \& Mills 1998, Wrobel 2000-2007).

A identificação das espécies é feita com base em características morfológicas, como a posição e proporção entre as fileiras de ctenos, dos canais meridionais e da faringe, além da presença ou ausência de tentáculos axiais, tentáculos orais, lobos, papilas, etc. 
As estruturas internas são facilmente observadas nesses animais de corpo translúcido.

\section{Resultados e Discussão}

Para o presente estudo, foram consideradas as 12 espécies planctônicas registradas para a plataforma continental brasileira (Mianzan 1999) e uma espécie bentônica registrada para o litoral norte do Estado de São Paulo (Marcus 1957, Oliveira \& Migotto 2007). Dentre as espécies planctônicas, cinco foram posteriormente registradas para o litoral norte do Estado de São Paulo (Oliveira \& Migotto 2006) e seis foram coletadas em áreas oceânicas nas regiões Norte e Nordeste do Brasil (Harbison et al. 1978). Embora os registros de Harbison et al. (1978) tenham sido realizados em áreas externas às águas territoriais brasileiras (até 200 milhas náuticas da costa, Zona Econômica Exclusiva), consideramos no presente estudo as espécies registradas para os pontos mais próximos das águas territoriais brasileiras, entre 200 e 300 milhas náuticas da costa, equivalente à Zona Econômica Exclusiva Expandida (Harbison et al. 1978, p. 243, pontos 540 a 550). No total, listamos 13 espécies para a costa brasileira (vide Tabela 1, Figuras 2-14).

Embora outras espécies nominais tenham sido registradas para águas costeiras e neríticas do Brasil (Tabela 2), elas não foram abordadas neste estudo por serem sinônimos de espécies listadas (vide Harbison \& Volovik, 1994 para sinônimos de Mnemiopsis leidyi).

Apesar da presente chave contemplar apenas as formas adultas, vale salientar que as formas larvais dos ctenóforos das ordens Lobata (vide Figura 15) muito se assemelham às formas adultas da ordem Cydippida, apresentando corpo de forma globosa e com longos tentáculos axiais ramificados. A diferenciação se dá pela orientação da bainha tentacular, voltada para a região oral nas larvas de ctenóforos lobados e para a região aboral nos ctenóforos da ordem Cydippida.
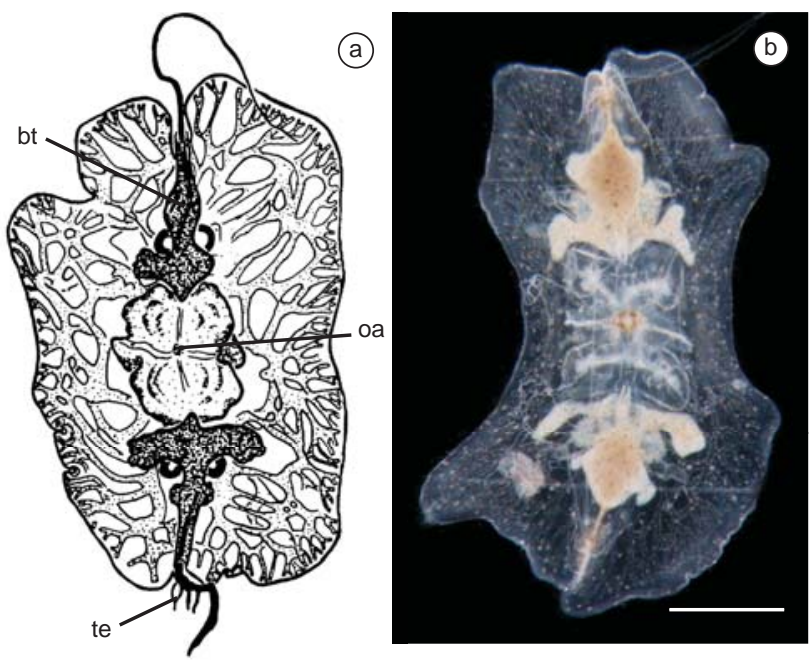

Figura 2. Vallicula multiformis Rankin, 1956. a) desenho em vista aboral (modificado de Rankin, 1956); e b) espécime em vista aboral (foto: A. Migotto). Legenda: bt, bainha tentacular; oa, órgão apical; te, tentilas. (Escala: $2 \mathrm{~mm}$ ).

Figure 2. Vallicula multiformis Rankin, 1956. a) drawing of the aboral view (after Rankin, 1956); and b) specimen in aboral view (photo: A. Migotto). Legend: bt, tentacular bulb, ao, apical organ; te, tentilla. (Scale: $2 \mathrm{~mm}$ ).

\section{Glossário dos Termos Utilizados no Estudo Morfológico dos Ctenophora}

Aurículas: Projeções corpóreas afiladas, posicionadas na extremidade oral das fileiras de ctenos subtentaculares, nos ctenóforos lobados. Aparentemente têm a função de promover a circulação de água próximo à boca, propiciando a captura de alimento pelos tentáculos orais. (Vide Figuras 9, 11 e 14.au)

Bainha tentacular: Estrutura que define o eixo tentacular, onde se aloja o tentáculo axial retraído. (Vide Figuras 1, 2, 6 e 15.bt)
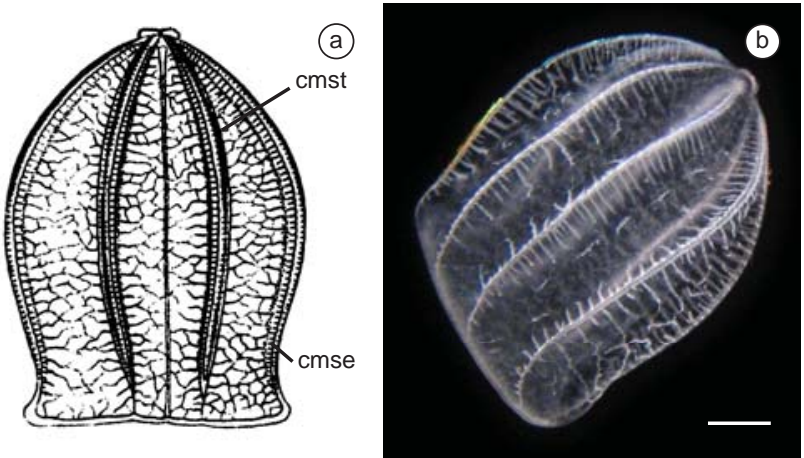

Figura 3. Beroe ovata Chamisso \& Eysenhardt, 1821. a) desenho em vista estomodeal (modificado de Mayer, 1912); e b) espécime em vista estomodeal (foto: O. Oliveira). Legenda: cmse, canal meridional subestomodeal; cmst, canal meridional subtentacular. (Escala: $1 \mathrm{~cm}$ ).

Figure 3. Beroe ovata Chamisso \& Eysenhardt, 1821. a) drawing of the stomodeal view (after Mayer, 1912); and b) specimen in stomodeal view (photo: O. Oliveira). Legend: cmse, substomodeal meridional canal; cmst, subtentacular meridional canal. (Scale: $1 \mathrm{~cm}$ ).
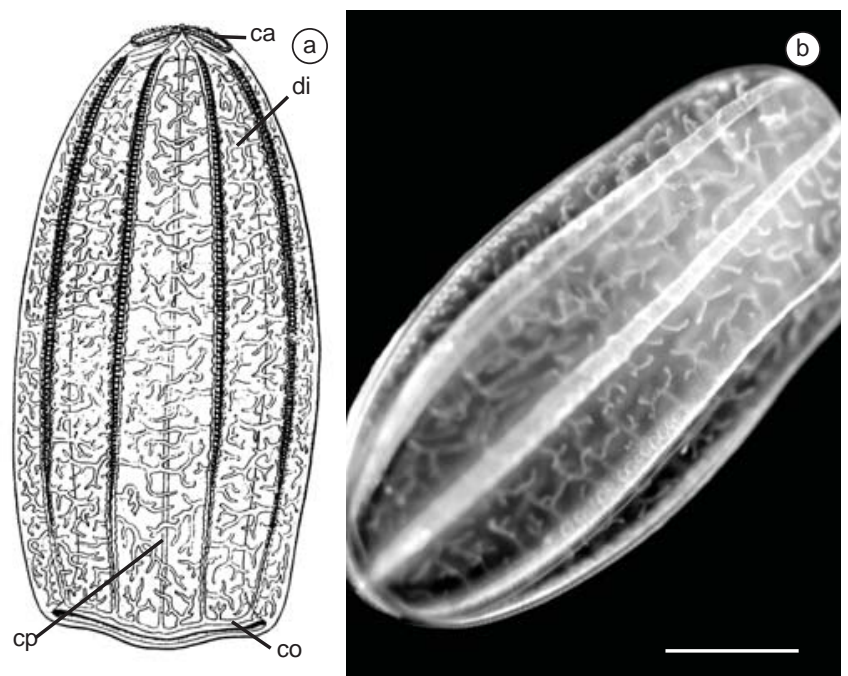

Figura 4. Beroe cucumis Fabricius, 1780. a) desenho em vista estomodeal (modificado de Mayer, 1912); and b) espécime em vista estomodeal (foto: S. Haddock). Legenda: ca, campo apical; co, canal oral; cp, canal paragástrico; di, divertículo. (Escala: $2 \mathrm{~cm}$ ).

Figure 4. Beroe cucumis Fabricius, 1780. a) drawing of the stomodeal view (after Mayer, 1912); b) specimen in stomodeal view (photo: S. Haddock). Legend: ca, polar field; co, oral canal; cp, paragastric canal; di, diverticulum. (Scale: $2 \mathrm{~cm}$ ). 

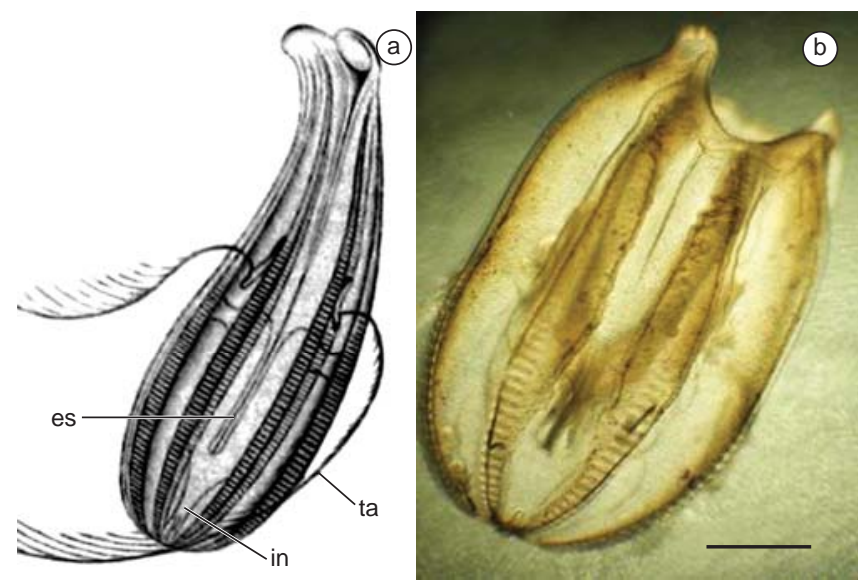

Figura 5. Lampea pancerina (Chun, 1879). a) desenho em vista tentacular (modificado de Chun, 1879); e b) espécime em vista estomodeal, com tentáculos axiais contraídos (foto: A. Moss). Legenda: es, estomodeu; in, infundíbulo; ta, tentáculo axial. (Escala: $1 \mathrm{~cm}$ ).

Figure 5. Lampea pancerina (Chun, 1879). a) drawing of the tentacular view (after Chun, 1979); and b) specimen in stomodeal view, with axial tentacles contracted (photo: A. Moss). Legend: es, stomodeum; in, infundibulum; ta, axial tentacle. (Scale: $1 \mathrm{~cm}$ ).

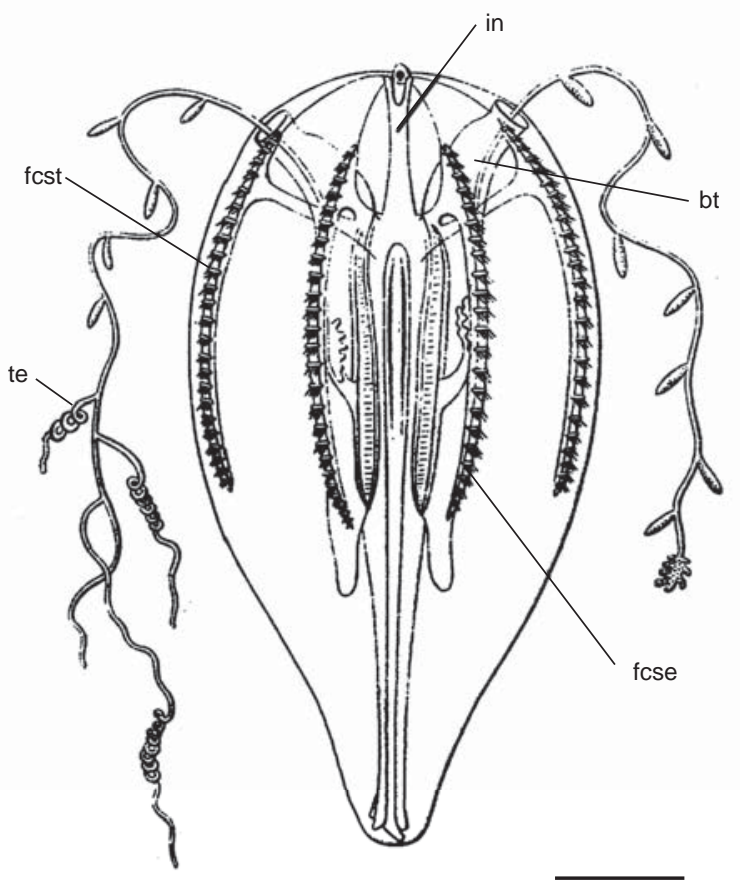

Figura 6. Hormiphora plumosa L.Agassiz, 1860. Desenho em vista tentacular (modificado de Mayer, 1912). Legenda: bt, bainha tentacular; fcse, fileira de ctenos subestomodeal; fcst, fileira de ctenos subtentacular; in, infundíbulo; te, tentila. (Escala: $3 \mathrm{~mm}$ ).

Figure 6. Hormiphora plumosa L.Agassiz, 1860. drawing of the tentacular view (after Mayer, 1912). Legend: bt, tentacular bulb; fcse, substomodeal ctene row; fcst, subtentacular ctene row; in, infundibulum; te, tentilla. (Scale: $3 \mathrm{~mm}$ ).
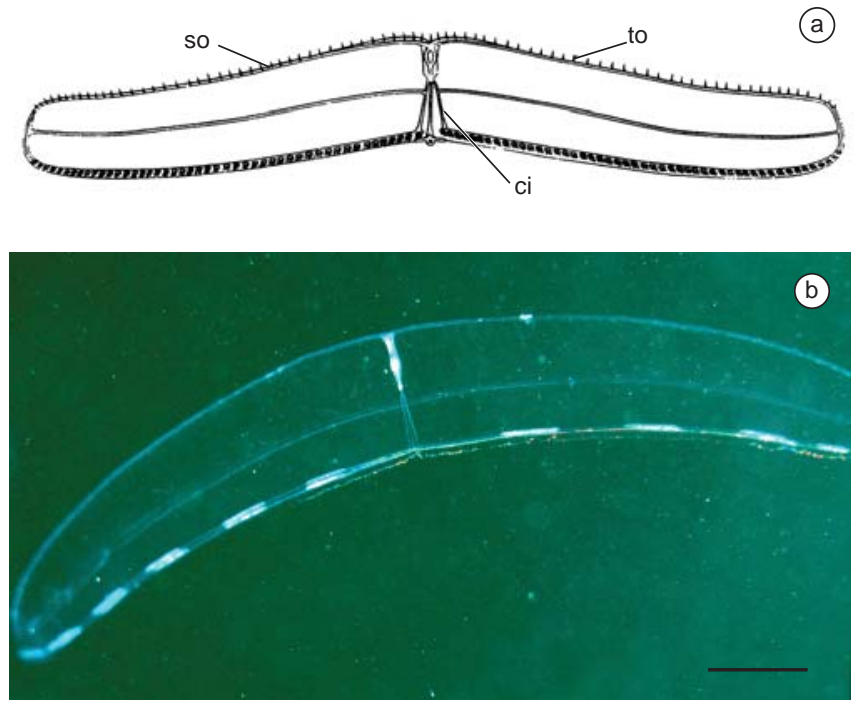

Figura 7. Velamen parallelum (Fol, 1869). a) desenho em vista estomodeal (modificado de Mayer, 1912); e b) espécime em vista estomodeal (foto: S. Haddock). Legenda: ci, canal interradial; so, sulco oral; to, tentáculos orais. (Escala: $2 \mathrm{~cm}$ ).

Figure 7. Velamen parallelum (Fol, 1869). a) drawing of the stomodeal view (after Mayer, 1912); and b) specimen in stomodeal view (photo: S. Haddock). Legend: ci, interadial canal; so, oral furrow; to, oral tentacles. (Scale: $2 \mathrm{~cm}$ )

(a)
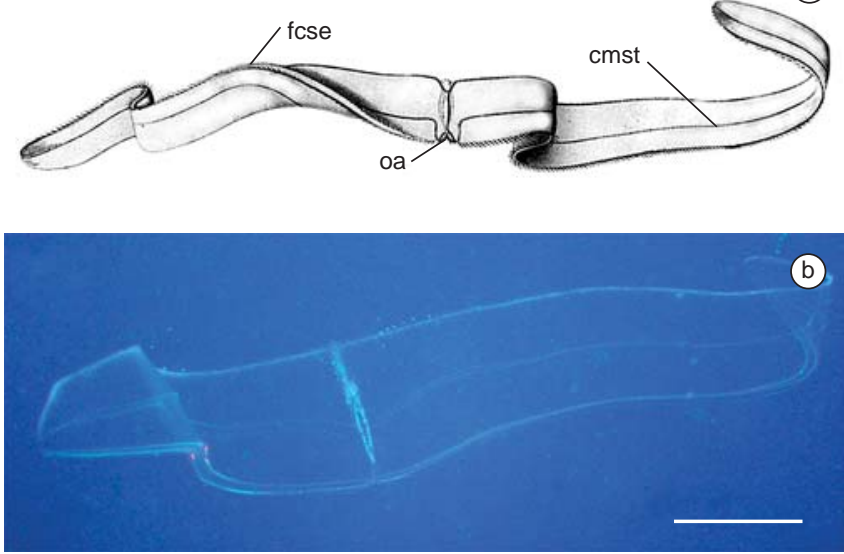

Figura 8. Cestum veneris Lesueur, 1813. a) desenho em vista estomodeal (modificado de Bigelow, 1904); e b) espécime em vista estomodeal (foto: A. Luca Jr). Legenda: cmst, canal meridional subtentacular; fcse, fileira de ctenos subestomodeal; oa, órgão apical. (Escala: $10 \mathrm{~cm}$ ).

Figure 8. Cestum veneris Lesueur, 1813. a) drawing of the stomodeal view (after Bigelow, 1904); and b) specimen in stomodeal view (photo: A. Luca Jr). Legend: cmst, subtentacular meridional canal; fcse, substomodeal ctene row; ao, apical organ. (Scale: $10 \mathrm{~cm}$ ).

Campo apical: Estrutura em forma de "8", localizada no pólo aboral, contendo o órgão apical ao centro, os poros anais e, por vezes, papilas sensoriais. (Vide Figura 4.ca).

Canal interradial: Canal que sai da região central do infundíbulo, em direção à região aboral, de forma oblíqua em relação aos eixos estomodeal e tentacular. (Vide Figura 7.ci). 
Canais meridionais: Tubos longitudinais que se ligam aboralmente ao estomodeu. Posicionados próximos às paredes corpóreas, proximalmente em relação às fileiras de ctenos.

Canais meridionais subestomodeais: Os quatro canais meridionais mais próximos ao plano de corte do eixo estomodeal. (Vide Figuras 3 e 13.cmse).

Canais meridionais subtentaculares: Os quatro canais meridionais mais próximos ao plano de corte do eixo tentacular. (Vide Figuras 3 e 8.cmst).

Canal oral: Canal que circunda a boca. (Vide Figura 4.co).

Canais paragástricos: Sulcos que correm longitudinalmente ao eixo tentacular do estomodeu. (Vide Figuras 4 e 10.cp).

Cidipídio: Fase larval, de corpo globoso e com dois longos tentáculos axiais, presente na maioria das espécies de ctenóforos (exceto os Beroida).

Coloblasto: Célula produtora de muco adesivo, presente nos tentáculos axiais e orais.

Ctenos: Placas formadas por cílios justapostos, cujo batimento é responsável pela locomoção nos ctenóforos planctônicos (= pentes ciliares)
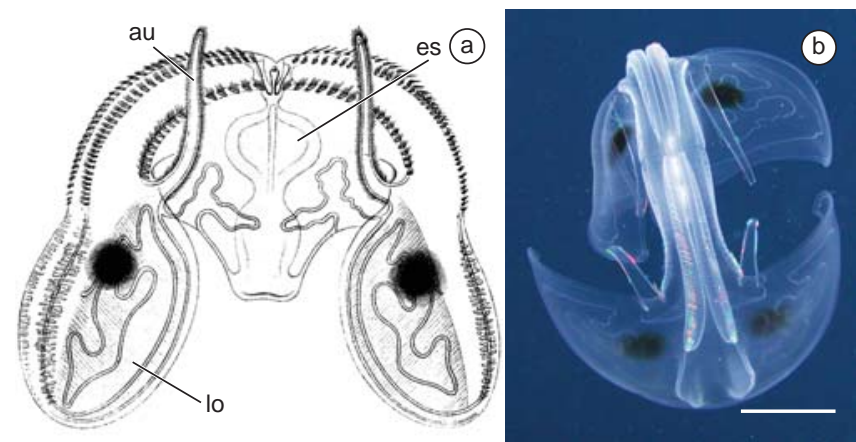

Figura 9. Ocyropsis maculata (Rang, 1828). a) desenho em vista estomodea (modificado de Mayer, 1912, fig. 11, como Ocyropsis crystallina); e b) espécime em vista aboral (foto: C. Dunn). Legenda: au, aurícula; es, estomodeu; lo, lobo oral. (Escala: $2 \mathrm{~cm}$ )

Figure 9. Ocyropsis maculata (Rang, 1828). a) drawing of the stomodeal view (after Mayer, 1912, fig. 11, as Ocyropsis crystallina); and b) specimen in aboral view (photo: C. Dunn). Legend: au, auricle; es, stomodeum; lo, oral lobe. (Scale: $2 \mathrm{~cm}$ )
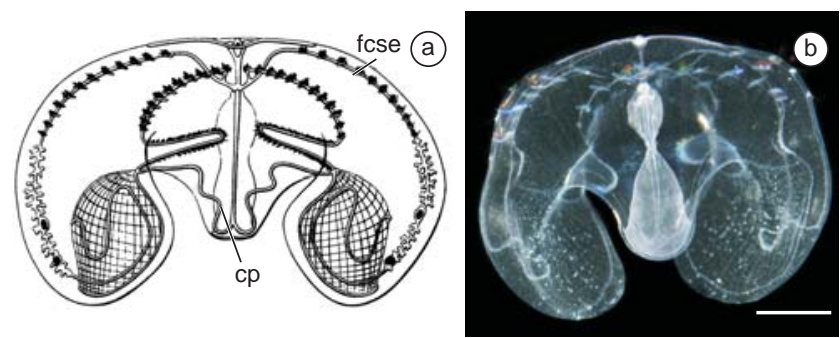

Figura 10. Ocyropsis crystallina (Rang, 1828). a) desenho em vista estomodeal (modificado de Mayer, 1912); e b) espécime em vista estomodeal (foto: A. Migotto). Legenda: cp, canal paragástrico; fcse, fileira de ctenos subestomodeal. (Escala: $2 \mathrm{~mm}$ ).

Figure 10. Ocyropsis crystallina (Rang, 1828). a) drawing of the stomodeal view (after Mayer, 1912); and b) specimen in stomodeal view (photo: A Migotto). Legend: cp, paragastric canal; fcse, subestomodeal ctene row. (Scale: $2 \mathrm{~mm}$ ).
Divertículo: Projeções laterais dos canais meridionais, responsáveis por um aumento de área do sistema gastrovascular. Presente nos Beroida. (Vide Figura 4.di).

Eixo estomodeal: Eixo de simetria que corta longitudinalmente o corpo no plano que atravessa o estomodeu em sua face mais achatada. (Vide Figura 1).
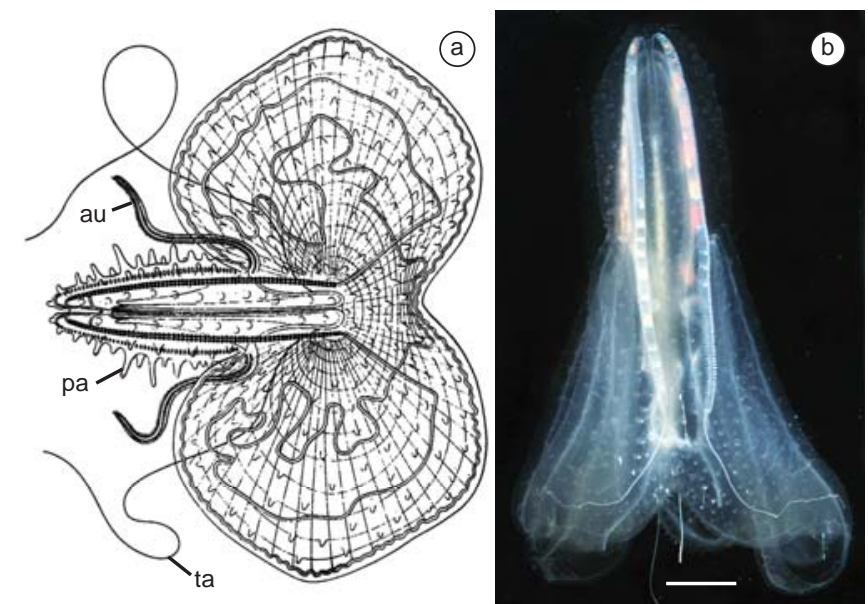

Figura 11. Leucothea multicornis (Quoy \& Gaimard, 1824). a) desenho em vista tentacular (modificado de Mayer, 1912, fig. 49, como Leucothea ochracea); e b) espécime em vista tentacular (Foto: A. Migotto). Legenda: au, aurícula; pa, papila; ta, tentáculo axial. (Escala: $2 \mathrm{~cm}$ ).

Figure 11. Leucothea multicornis (Quoy \& Gaimard, 1824). A, drawing of the tentacular view (modifyed from Mayer, 1912, fig. 49, as Leucothea ochracea); and b) specimen in tentacular view (photo: A. Migotto). Legend: au, auricle; pa, papilla; ta, axial tentacle. (Scale: $2 \mathrm{~cm}$ ).

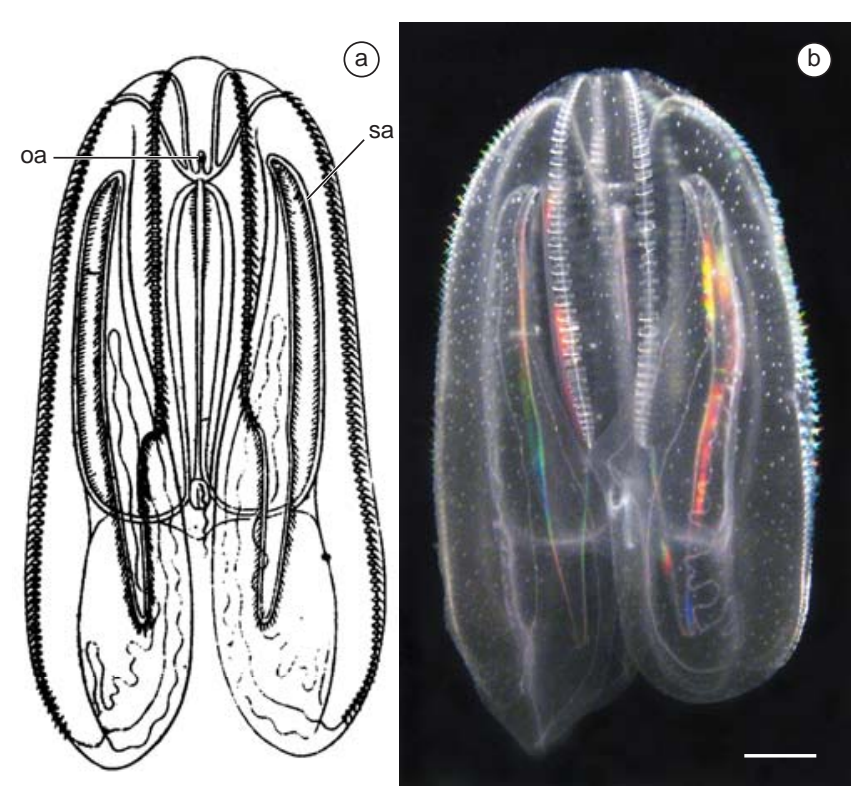

Figura 12. Mnemiopsis leidyi A.Agassiz, 1865. a) desenho em vista estomodeal (modificado de Mayer, 1912); e b) espécime em vista estomodeal (foto: O. Oliveira). Legenda: oa, órgão apical; sa, sulco auricular. (Escala: $1 \mathrm{~cm}$ ).

Figure 12. Mnemiopsis leidyi A.Agassiz, 1865. a) drawing of the stomodeal view (after Mayer, 1912); and b) specimen in stomodeal view (photo: O. Oliveira). Legend: ao, apical organ; sa, auricular furrow. (Scale: $1 \mathrm{~cm}$ ). 


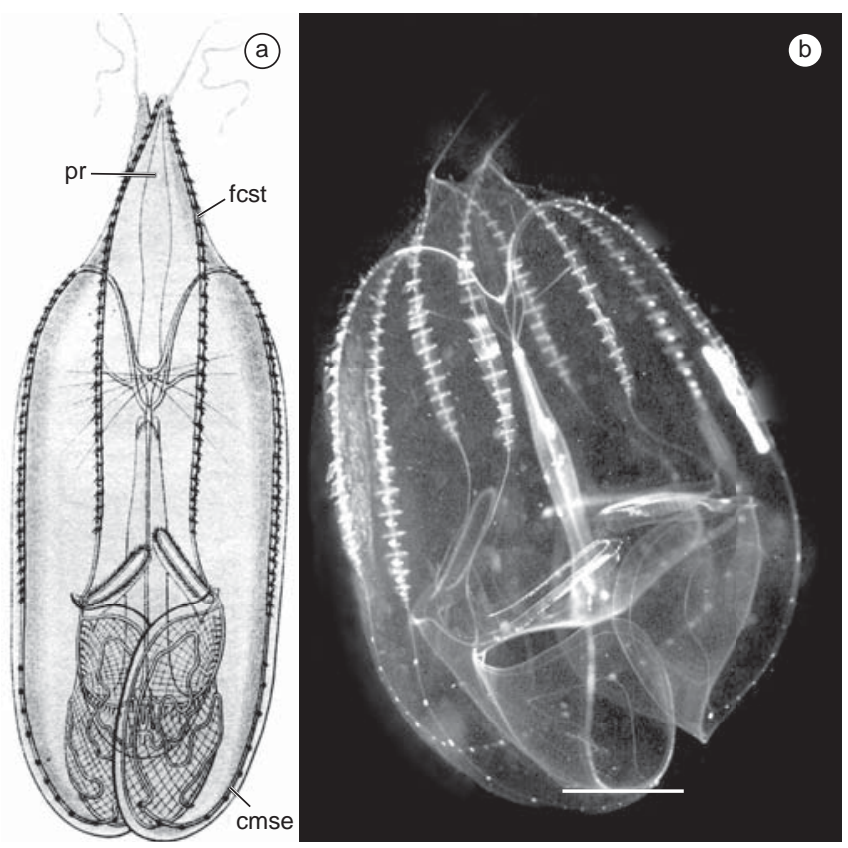

Figura 13. Eurhamphaea vexilligera Gegenbaur, 1856. a) desenho em vista estomodeal (modificado de Mayer, 1912); e b) espécime em vista estomodeal (foto: S. Haddock). Legenda: cmse, canal meridional subestomodeal; fcst, fileira de ctenos subtentacular; pr, projeção apical. (Escala: $1 \mathrm{~cm}$ ).

Figure 13. Eurhamphaea vexilligera Gegenbaur, 1856. a) drawing of the stomodeal view (after Mayer, 1912); and b) specimen in stomodeal view (photo: S. Haddock). Legend: cmse, substomodeal meridional canal; fcst, subtentacular ctene row; pr, apical projection. (Scale: $1 \mathrm{~cm}$ ).

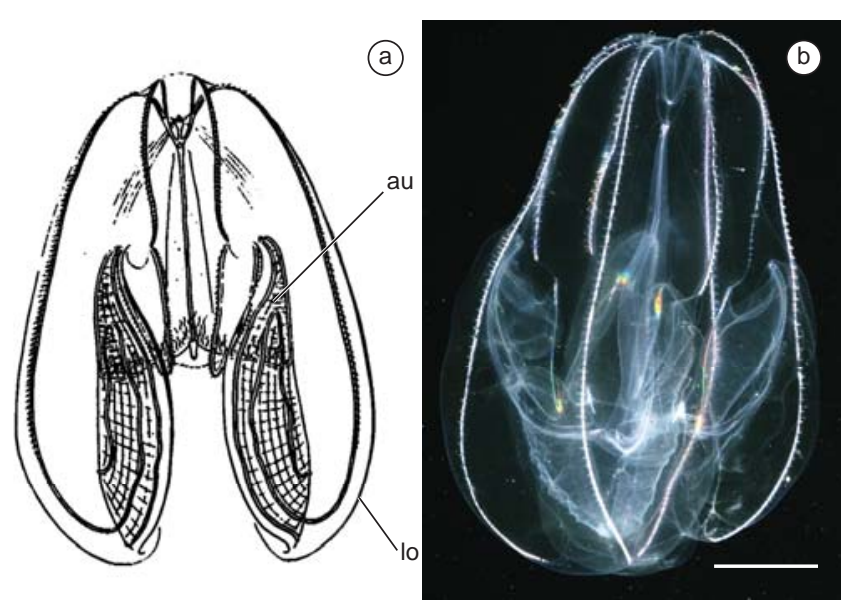

Figura 14. Bolinopsis vitrea (L.Agassiz, 1860). a) desenho em vista estomodeal (modificado de Mayer, 1912); e b) espécime em vista estomodeal (foto: A. Migotto). Legenda: au, aurícula; lo, lobo oral. (Escala: $1 \mathrm{~cm}$ ).

Figure 14. Bolinopsis vitrea (L.Agassiz, 1860). a) drawing of the stomodeal view (after Mayer, 1912); and b) specimen in stomodeal view (photo: A. Migotto). Legend: au, auricle; lo, oral lobe. (Scale: $1 \mathrm{~cm}$ ).

Eixo tentacular: Eixo de simetria que corta longitudinalmente o corpo no plano que atravessa as bainhas tentaculares. (Vide Figura 1).

Estomodeu: Estrutura que define o eixo estomodeal. Porção achatada e alongada do infundíbulo onde ocorre a digestão do alimento (= "faringe"). (Vide Figuras 1, 5 e 9.es).

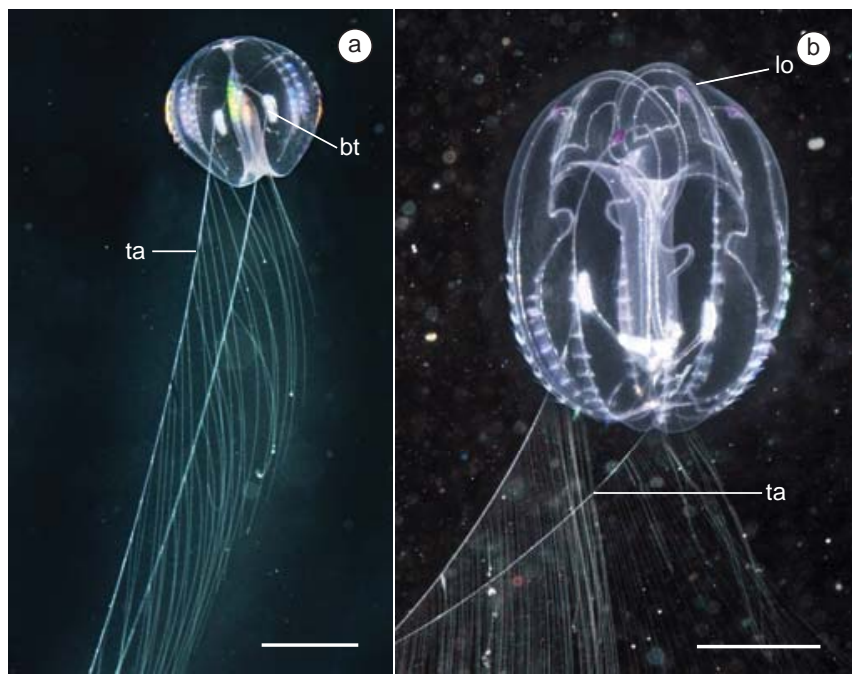

Figura 15. Formas larvais de espécies da ordem Lobata. a) larva de Mnemiopsis leidyi (foto: A. Migotto); e b) larva de Bolinopsis vitrea em fase de metamorfose (foto: A. Migotto). Legenda: bt, bainha tentacular; lo, lobo oral; ta, tentáculo axial. (Escala: $5 \mathrm{~mm}$ ).

Figure 15. Larvae of lobate ctenophores. a) larva of Mnemiopsis leidyi (photo: A. Migotto); and b) larva of Bolinopsis vitrea in metamorphosis (photo: A. Migotto). Legend: bt, tentacular bulb; lo, oral lobe; ta, axial tentacle. (Scale: $5 \mathrm{~mm})$.

Fileira de ctenos: Sequiências longitudinais de pentes ciliares, localizadas na epiderme dos animais, distalmente em relação aos canais meridionais.

Fileiras de ctenos subestomodeal: As quatro fileiras de pentes ciliares mais próximos ao plano de corte do eixo estomodeal. (Vide Figuras 6, 8 e 10.fcse).

Fileiras de ctenos subtentaculares: As quatro fileiras de pentes ciliares mais próximos ao plano de corte do eixo tentacular. (Vide Figuras 6 e 13.fcst).

Hemisférios: Metades iguais do corpo do ctenóforo quando dividido nos eixos estomodeal ou tentacular.

Infundíbulo: Tubo central do organismo, prolongando-se da boca à região aboral. Principal cavidade do sistema gastrovascular. (Vide Figura 6.in).

Lobos orais: Prolongações corpóreas na região oral dos hemisférios divididos pelo plano de corte tentacular, presentes nos ctenóforos lobados. (Vide Figuras 9, 14 e 15.lo).

Órgão apical: Estrutura sensorial, posicionada no centro do campo apical, composta por um estatocisto. (Vide Figuras 2, 8 e 12.oa)

Papila: Saliência ou projeção na epiderme, com funções supostamente sensoriais. (Vide Figura 11.pa).

Pentes ciliares: Vide "ctenos".

Pólo aboral: Extremidade do corpo oposta a posição da boca.

Poro anal: Estrutura localizada lateralmente ao órgão apical, supostamente responsável pela liberação parcial de resíduos metabólicos e da digestão.

Projeção apical: Projeção do corpo na região aboral. (Vide Figura 13.pr).

Sistema gastrovascular: Conjunto de tubos no interior do organismo, pelos quais ocorre o transporte de nutrientes resultantes da digestão extracelular, excretas e gametas. Inclui o infundíbulo e os canais.

Sulco auricular: Reentrância que se alonga da região oral até a base dos lobos em parte dos ctenóforos lobados. Recoberta por tentáculos orais. (Vide Figura 12.sa). 
Tabela 2. Espécies nominais excluídas da chave por se tratarem de possíveis sinonímias.

Table 2. Species names recorded for the Brazilian coast, but excluded from the key as possible synonyms.

\begin{tabular}{llll}
\hline \multicolumn{1}{c}{ Espécie nominal } & \multicolumn{1}{c}{ Registro } & \multicolumn{1}{c}{ Local } & Sinônimo \\
\hline Mnemia schweiggeri & Eschscholtz 1825, 1829 & Rio de Janeiro & Mnemiopsis leidyi A.Agassiz, 1865 \\
Alcinoe vermiculata & Rang 1828 & Rio de Janeiro & Mnemiopsis leidyi A.Agassiz, 1865 \\
Mnemiopsis mccradyi & Petrechen 1946 & Santos & Mnemiopsis leidyi A.Agassiz, 1865 \\
Beroe gilva & Eschscholtz 1829 & Costa brasileira & Beroe ovata Chamisso \& Eysenhardt, 1821 \\
\hline
\end{tabular}

Sulco oral: Reentrância recoberta por tentáculos orais, disposta ao longo de toda a margem oral dos ctenóforos cestídeos. (Vide Figura 7.so).

Tentáculo axial: Estrutura alongada e por vezes ramificada, originada na bainha tentacular. (Vide Figuras 5, 11 e 15.ta).

Tentáculos orais: Filamentos dispostos ao longo da margem oral de alguns ctenóforos, dotados de coloblastos e responsáveis pela captura e transporte das presas para a boca. (Vide Figura 7.to).

Tentilas: Filamentos dos tentáculos axiais. Contêm coloblastos, responsáveis pela captura de presas por adesão. (Vide Figuras 2 e 6.te).

\section{Agradecimentos}

Os autores são gratos aos Drs. Anthony Moss (Auburn University, EUA), Casey Dunn (University of Hawaii, EUA) e Steven Haddock (MBARI, EUA) e ao Sr. Armando de Luca Jr (Nautilus Dive Center, Santos) pela gentileza em permitir o uso de suas fotos no presente estudo; aos dois revisores anônimos por suas valiosas sugestões; ao Centro de Biologia Marinha da Universidade de São Paulo, pelo apoio e uso de sua infra-estrutura. Este estudo foi financiado com recursos da FAPESP (Proc. 2004/15300-0; 2004/09961-4) e CNPq (55.7333/2005-9, 490348/2006-8, 305735/2006-3).

\section{Chave de Identificação para as Espécies de Ctenóforos da Costa Brasileira}

1 Forma bentônica, rastejante. Fileiras de ctenos ausentes no adulto. (Ordem Platyctenida)..... Vallicula multiformis (Fig. 2) (vide Rankin 1956, p. 68-69, Figuras 1-8, pranchas 2-3; Marcus 1957, p. 87-89, prancha 1; Oliveira \& Migotto 2007)

- Forma planctônica, livre-natante. Fileiras de ctenos presentes no adulto.....

2 Tentáculos axiais ausentes nas formas larvais e adultas. Boca larga, ocupando toda a extremidade oral do corpo e faringe alargada em toda a extensão do corpo. (Ordem Beroida).

- Tentáculos axiais presentes ao menos nas formas larvais. Boca se restringindo à região central, não necessariamente posicionada na extremidade oral, e faringe achatada, restrita à região central do corpo

3 Corpo achatado no eixo tentacular. Divertículos gastrovasculares oriundos de canais meridionais adjacentes se intercomunicando, podendo formar anastomoses ................................................................................................................................... Beroe ovata (Fig. 3) (vide Mayer 1912, p. 49-52, pranchas 15-16; Mianzan 1999, p. 569, figura 3.17; Oliveira \& Migotto 2006, p. 17-22, Figura 6)

- Corpo cilíndrico. Divertículos gastrovasculares nunca se comunicando. Beroe cucumis (Fig. 4) (vide Mayer 1912, p. 52-55 e pranchas 15 e 17; Mianzan 1999, p. 569, figura 3.16)

4 Corpo esférico ou ovalado, não apresentando projeções laterais. Bainha tentacular orientada para a porção aboral. (Ordem Cydippida)..... 5 - Corpo de forma variável, achatado no eixo tentacular, apresentando projeções laterais. Bainha tentacular orientada para a porção oral..6

5 Região oral alargada, com boca expansível e estomodeu eversível. Abertura da bainha tentacular no hemisfério oral do corpo, na altura da extremidade oral das fileiras de ctenos subtentaculares. (vide Mianzan 1999, p. 567, figura 3.6) Lampea pancerina (Fig. 5)

- Região oral afilada, com estomodeu não eversível. Abertura da bainha tentacular no hemisfério aboral do corpo, próximo à extremidade aboral das fileiras de ctenos subtentaculares Hormiphora plumosa (Fig. 6) (vide Mayer 1912, p. 15-16, prancha 2; Mianzan 1999, p. 567, figura 3.4)

6 Projeções laterais do corpo alongadas no eixo estomodeal, conferindo ao corpo forma de fita. Fileiras de ctenos tentaculares pelo menos dez vezes menores que as fileiras de ctenos estomodeais. (Ordem Cestida)......

- Projeções laterais do corpo em forma de lobos orientados em direção à porção oral. Fileiras de ctenos tentaculares com cerca de metade do comprimento das fileiras de ctenos estomodeais. (Ordem Lobata).

7 Canais subtentaculares conectados aos canais interradiais a meia distância entre as extremidades oral e aboral. Extremidades das projeções laterais afiladas. Corpo atingindo até $30 \mathrm{~cm}$ de comprimento no eixo estomodeal....................................... Velamen parallelum (Fig. 7) (vide Mayer 1912, p. 46-47, pranchas 13-14, como Folia parallela; Mianzan 1999, p. 569, Figura 3.15) 
- Canais subtentaculares conectados aos canais interradiais próximo à região aboral. Extremidades das projeções laterais arredondadas. Corpo atingindo até $200 \mathrm{~cm}$ no eixo estomodeal ............................................................................................. Cestum veneris (Fig. 8) (vide Mayer 1912, p. 44-46, prancha 12; Mianzan 1999, p. 568, Figura 3.14)

8 Ausência de tentáculos orais. Órgão apical demarcando a extremidade aboral 9

- Presença de tentáculos orais. Órgão apical situado em depressão proximal à extremidade aboral

9 Presença de mancha de pigmentação escura em cada lobo... (vide Mayer 1912, p. 40; Mianzan 1999, p.568, Figura 3.12)

- Ausência de mancha de pigmentação escura em cada lobo (vide Mayer 1912, p. 38-39, prancha 10; Mianzan 1999, p. 568, figura 3.11; Oliveira \& Migotto 2006, p. 15-17, Figura 5)

10 Superfície corporal recoberta por papilas retráteis. Presença de longos tentáculos axiais não ramificados no adulto (vide Mianzan 1999, p. 568, figura 3.10; Oliveira \& Migotto 2006, p.12-14, Figura 4)

Leucothea multicornis (Fig. 11)

- Superfície corporal lisa ou apresentando pequenas verrugas não retráteis. Tentáculos axiais ausentes ou vestigiais no adulto

11 Lobos surgindo na altura do órgão apical, formando extenso sulco auricular entre o lobo e a região central do corpo ...................................................................................................................................................... Mnemiopsis leidyi (Fig. 12) (vide Mayer 1912, p. 26-34, pranchas 6-8, como M. leidyi, M. mccradyi e M. gardeni; Petrechen 1946, p. 117-118, figuras 1-2, como M. mccradyi; Mianzan 1999, p. 568, Figuras 3.8-3.9, como M. leidyi e M. maccradyi; Oliveira \& Migotto 2006, p. 8-12, Figura 3)

- Lobos surgindo na altura da base das aurículas, formando curto sulco auricular próximo à região oral.

12 Presença de projeções apicais triangulares na extremidade aboral (vide Mayer 1912, p. 41-42, prancha 11; Mianzan 1999, p. 568, figura 3.13)

- Ausência de projeções apicais

Eurhamphaea vexilligera (Fig. 13) (vide Mayer 1912, p. 22-26, prancha 5; Mianzan 1999, p. 568, figura 3.7; Oliveira \& Migotto 2006, p. 4-7, Figura 2)

\section{Referências Bibliográficas}

ADAMS, H.R., FLERCHINGER, A.P. \& STEEDMAN, H.F. 1976. Ctenophora fixation and preservation. In Zooplankton fixation and preservation (H.F. Steedman, ed.). Unesco Press, Paris, p. 270-271.

AGASSIZ, L. 1860. Contributions to the natural history of the United States of America, Vol. 3. Little, Brown and Co., Boston, p. 1-301.

BAKER, L.D. \& REEVE, M.R. 1974. Laboratory culture of the lobate ctenophore Mnemiopsis mccradyi with notes on feeding and fecundity. Mar. Biol. 26:57-62.

BIGELOW, H.B. 1904. Medusae from the Maldive Islands. Bull. Mus. Comp. Zool. Harvard Coll. 39(9):244-269.

BISHOP, J.W. 1968. A comparative study of feeding rates of tentaculate ctenophores. Ecology 49(5):996-997.

BOERSMA, M., MALZAHAN, A.M., GREVE, W. \& JAVIDPOUR, J. 2007. The first occurrence of the ctenophore Mnemiopsis leidyi in the North Sea. Helgol. Mar. Res. 61:153-155.

BRUSCA, R.C. \& BRUSCA, G.J. 2007. Invertebrados. 2 ed. Guanabara Koogan, Rio de Janeiro.

CHUN, C. 1879. Die im Golf von Neapel erscheinenden Rippenquallen. Mittelmeer Zoologischen Station von Neapel zugleich repert Mittelmeerkd $1: 180-217$.

CORRÊA, D.D. 1987. Ctenophora. In Manual de técnicas para a preparação de coleções zoológicas. Sociedade Brasileira de Zoologia, Curitiba, p. $195-199$.

ESCHSCHOLTZ, J.F. 1825. Bericht über die zoologische Ausbeute während der Reise von Kronstadt bis St. Peter- und Paul. Isis 1:733-747.

ESCHSCHOLTZ, J.F. 1829. System der Acalephen. F. Dümmler, Berlin.

GESAMP (IMO/FAO/UNESCO-IOC/WMO/WHO/IAEA/UN/UNEP JOINT GROUP OF EXPERTS ON THE SCIENTIFIC ASPECTS OF MARINE ENVIRONMENTAL PROTECTION), 1997. Opportunistic settlers and the problem of the ctenophore Mnemiopsis leidyi invasion in the Black Sea. GESAMP Reps. Stud. 58:1-84.
HADDOCK, S.H.D. 2004. A golden age of gelata: past and future research on planktonic ctenophores and cnidarians. Hydrobiologia 530/531:549-556.

HADDOCK, S.H.D. \& CASE, J.F. 1995. Not all ctenophores are bioluminescent: Pleurobrachia. Biol. Bull. 189:356-362.

HADDOCK, S.H.D. \& HEINE, J.N. 2005. Scientific blue-water diving. California Sea Grant, La Jolla, California.

HARBISON, G.R. \& MADIN, L.P. 1982. Ctenophora. In Synopsis and classification of living organisms (S.P. Parker, ed.). Vol. 1. McGraw-Hill, New York, p. 707-715.

HARBISON, G.R. \& MILLER, R.L. 1986. Not all ctenophores are hermaphrodites. Studies on the systematics, distribution, sexuality and development of two species of Ocyropsis. Mar. Biol. 90:413-424.

HARBISON, G.R., MADIN, L.P. \& SWANBERG, N.R. 1978. On the natural history and distribution of oceanic ctenophores. Deep-Sea Res. 25:233-256

HARBISON, G.R. \& VOLOVIK, S.P. 1994. The ctenophore, Mnemiopsis leidyi, in the Black Sea: a holoplanktonic organism transported in the ballast of ships. In: Non-Indigenous estuarine \& marine organisms (NEMO) and introduced marine species. Proceedings of the Conference and Workshop, NOAA Technological Report, U.S. Department of Commerce. U.S. Government Printing Office, Washington, p. 25-36.

HYMAN, L.H. 1940. Invertebrates: Protozoa through Ctenophora. McGraw-Hill, New York.

KIDEYS, A.E. 2002. Fall and rise of the Black Sea ecosystem. Science 297:1482-1483.

MARCUS, E.B.R. 1957. Vallicula multiformis Rankin, 1956, from Brazil. Bolm. Inst. Oceanogr. 7(1-2):87-91.

MAYER, A.G. 1912. Ctenophores of the Atlantic Coast of North America. Public. Carnegie Inst. Wash., 162:1-58.

MIANZAN, H.W. 1999. Ctenophora. In South Atlantic Zooplankton (D. Boltovskoy, ed.). Backhuys Publishers. p. 561-573. 
MILLS, C.E. INTERNET 1998-2007. Phylum Ctenophora: list of all valid species names. Documento eletrônico da Internet, disponível em http:// faculty.washington.edu/cemills/Ctenolist.html. Publicado pelo autor em março de 1998, última atualização em 13 de maio de 2007. (Último acesso em 31 de maio de 2007).

MILLS, C.E. \& MILLER, R.L. 1984. Ingestion of a medusa (Aegina citrea) by the nematocyst-containing ctenophore (Haeckelia rubra, formerly Euchlora rubra): phylogenetic implications. Mar. Biol. 78:215-221.

NIELSEN, C. 2001. Animal evolution: interrelationships of the living phyla. Oxford University Press, Oxford.

OLIVEIRA, O.M.P. 2007. The presence of the ctenophore Mnemiopsis leidyi in the Oslofjorden and considerations on the initial invasion pathways to the North and Baltic Seas. Aquat. Invasions 2(3):185-189.

OLIVEIRA, O.M.P. \& MARQUES, A.C. 2007. Geographic distribution and predatory impact of the ctenophore Mnemiopsis leidyi: a potential threat to the fisheries and planktonic ecosystems from Brazilian coast? In Plankton Symposium IV Abstracts (M.J. Pereira et al., eds.). BDUA J. Biol. 2:252.

OLIVEIRA, O.M.P. \& MIGOTTO, A.E. 2006. Pelagic ctenophores from the São Sebastião Channel, southeastern Brazil. Zootaxa 1183:1-26.

OLIVEIRA, O.M.P. \& MIGOTTO, A.E. 2007. Que bicho estranho! Animal marinho de morfologia aberrante é encontrado no litoral paulista. Ciên. Hoje 40(236):72-73.

PETRECHEN, M.A. 1946. Ocorrência de Mnemiopsis mccradyi, Mayer 1900 (Ctenophora) no litoral de São Paulo. An. Acad. Bras. Ciên. 18(2):117-120.
PODAR, M., HADDOCK, S.H.D., SOGIN, M.L. \& HARBISON, G.R. 2001. A molecular phylogenetic framework for the phylum Ctenophora using 18S rRNA genes. Mol. Phylogen. Evol. 21(2):218-230.

RANG, P.C.A.L. 1828. Établissement de la famille des Bérö̈des dans l'ordre des acalèphes libres, et description de deux genres nouveaux qui lui appartiennent. Mem. Soc. Hist. Nat. Paris. 4:166-173.

RANKIN, J.J. 1956. The structure and biology of Vallicula multiformis, gen. et sp. nov., a plactyctenid ctenophore. J. Linn. Soc. London. 43:55-71.

RASKOFF, K.A., SOMMER, F.A., HAMNER, W.M. \& CROSS, K.M. 2003. Collection and culture techniques for gelatinous zooplankton. Biol. Bull. 204:68-80.

SWANBERG, N. 1974. The feeding behavior of Beroe ovata. Mar. Biol. 24:69-76.

WALLBERG, A., THOLLESSON, M., FARRIS, J.S. \& JONDELIUS, U. 2004. The phylogenetic position of the comb jellies (Ctenophora) and the importance of taxonomic sampling. Cladistics 20:558-578.

WELCH, V.L., VIGNERON, J.P. \& PARKER, A.R. 2005. The cause of colouration in the ctenophore Beroe cucumis. Curr. Biol. 15(24):R985-R986.

WROBEL, D. INTERNET 2000-2007. Photographing Gelatinous Zooplankton. Documento eletrônico da Internet, disponível em http://jellieszone. com/photography.htm. (Acessado em 31 de maio de 2007).

WROBEL, D. \& MILLS, C.E. 1998. Pacific coast pelagic invertebrates. A guide to the common gelatinous animals. Sea Challengers \& Monterey Bay Aquarium, Monterey.

ZRZAVÝ, J., MIHULKA, S., KEPKA, P., BEZDEK, A. \& TIETZ, D. 1998. Phylogeny of the Metazoa based on morphological and 18S ribosomal DNA evidence. Cladistics 14:249-285. 